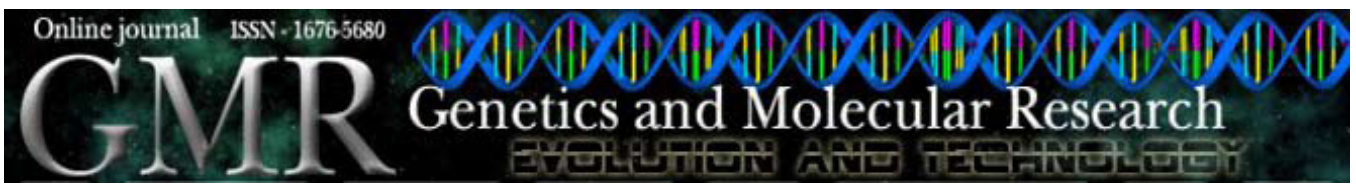

\title{
Rapid determination of transgene copy number in tobacco by competitive PCR using a pair of SSR primers
}

\author{
K.Y. Xu ${ }^{1}$, S.H. Wang ${ }^{1}$, L. Xi ${ }^{1,2}$, Q.J. Wang ${ }^{1,3}$, C. Dong ${ }^{1,4}$, J.Y. Zhang ${ }^{1}$, \\ S.C. Qu ${ }^{1}$ and Z. Zhang ${ }^{1}$ \\ ${ }^{1}$ College of Horticulture, Nanjing Agricultural University, \\ Nanjing, Jiangsu, China \\ ${ }^{2}$ Department of Wood Sciences, University of British Columbia, Vancouver, \\ British Columbia, Canada \\ ${ }^{3}$ Liaoning Agricultural Vocation-Technical College, Yingkou, Liaoning, China \\ ${ }^{4}$ Department of Horticulture, Heilongjiang Academy of Agricultural Sciences, \\ Haerbin, Heilongjiang, China
}

Corresponding author: Z. Zhang

E-mail: zhangzh@njau.edu.cn / qscnj@njau.edu.cn

Genet. Mol. Res. 9 (2): 935-940 (2010)

Received January 28, 2010

Accepted March 15, 2010

Published May 18, 2010

DOI 10.4238/vol9-2gmr790

\begin{abstract}
We developed a straightforward, rapid, and inexpensive method to determine transgene copy number in tobacco. The plasmid (pSSRCopy) used for tobacco transformation contains a simple sequence repeat (SSR) locus, PT1199, which was partially deleted in the middle, a homogenous SSR locus in tobacco K326. A 168-bp segment of the cloned PT1199 was shortened to 95 bp by deleting a 73-bp internal fragment. Using a pair of SSR primers, competitive PCR was amplified from genomic DNA from transgenic tobacco harboring pSSRCopy, and the two expected bands were found. The 168-bp band (SSR-168) corresponds to endogenous PT1199 and the 95-bp band (SSR-95) comes from the integrated pSSRCopy. A single
\end{abstract}


copy of a transgene can be easily distinguished from multiple copies by comparing band densities.

Key words: Copy number; Transgene; Competitive PCR; SSR; Tobacco

\section{INTRODUCTION}

Tobacco is a model plant for the study of transgene expression, and also, it is a very important economic crop worldwide. Genetic modification has been exploited far and wide to study the biology and development of plants as well as to improve commercial crops with fine agronomic characters (Mason et al., 2002). The transgenic plants with a single transgene copy are gladly accepted because multiple copies often bring on gene silencing (Kumpatla et al., 1997). The determination of transgene copy numbers in tobacco is time-consuming. Southern blotting hybridization is the most common method for copy number determination, but it is time-consuming and labor-intensive. Some investigators have attempted to simplify the method for copy number determination using real-time polymerase chain reaction (PCR) (Yang et al., 2005; Yi et al., 2008). This is the most accurate method to determine transgene copy number. However, this method needs expensive instrumentation, such as the Corbett Research Rotor-Gene 3000, and requires complicated calculations to determine copy number. In this study, we developed a rapid, straightforward, and inexpensive method to determine transgene copy number in tobacco. Competitive PCR was performed using a pair of simple sequence repeat (SSR) primers. The copy number of the exogenous gene in transgenic tobacco can be determined according to the intensities of endogenous and exogenous bands in ethidium bromide-stained gels. To confirm reliability, the results of this method were compared with the results obtained with Southern blotting. We conclude that our method is reliable for distinguishing single from multiple transgene copies.

\section{MATERIAL AND METHODS}

\section{Plasmid construction and genetic transformation}

A homogenous SSR locus PT1199 was found in tobacco K326, and a pair of SSR primers was obtained as follows: sense primer, 5'-TCGGGTCGTTACAACTGATG-3'; antisense primer, 5'-CCACGTCATTCGGAGTTGTT-3' (Bindler et al., 2007). Using the primers obtained, SSR-168 was amplified from the genomic DNA of tobacco K326 in a PTC100 Peltier Thermal Cycler (MJ Research Bio-Rad, USA) with an overlay of mineral oil. The SSR-95 with an HindIII site and an SphI site at each end, resulting from the deletion of a 73-bp internal fragment from the SSR-168, was synthesized by GenScript Technology Co., Ltd. (Nanjing, China). The chemosynthetic SSR-95 was subcloned into the HindIII/SphI site of the pCAMBIA-1301 plasmid. The plasmid pSSRCopy (Figure 1) was constructed as in the above-mentioned methods. This plasmid was introduced into the Agrobacterium tumefaciens strain EHA105, according to the freeze-thaw method (Hofgen and Willmitzer, 1988). Four transgenic tobacco lines (A, B, C, D) were generated by the leaf disc method (Horsch et al., 1985 ) and used in determining transgene copy number. 


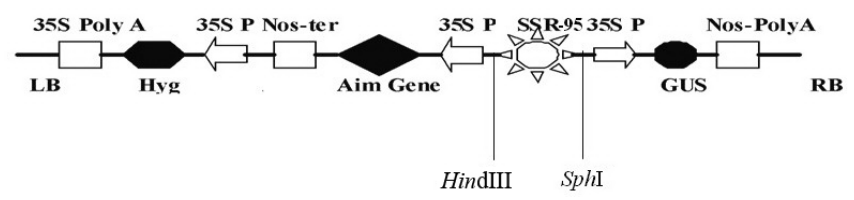

Figure 1. T-border region of plasmid pSSRCopy. SSR-95 = a 95-bp DNA obtained by deleting a 73-bp internal fragment from a 168-bp segment of the cloned simple sequence repeat (SSR) locus PT1199 of tobacco K326; $35 \mathrm{~S} \mathrm{P}=$ cauliflower mosaic virus $35 \mathrm{~S}$ promoter driving the expression of hygromycin phosphotransferase gene (Hyg) and beta-D-glucuronidase gene (GUS); the backbone of this plasmid is pCAMBIA-1301.

\section{Competitive PCR}

Genomic DNA was isolated from tobacco leaf material of 8-week-old transgenic plants by the CTAB method (Murray and Thompson, 1980). The standard PCR mixture contained 50 ng genomic DNA, $0.5 \mu \mathrm{M}$ of each primer, $50 \mu \mathrm{M}$ of each dNTP (dATP, dGTP, dTTP, dCTP), $20 \mathrm{mM}$ Tris- $\mathrm{HCl}, \mathrm{pH}$ 8.4, $20 \mathrm{mM} \mathrm{KCl}, 10 \mathrm{mM}\left(\mathrm{NH}_{4}\right)_{2} \mathrm{SO}_{4}, 2 \mathrm{mM} \mathrm{MgSO}_{4}$ and $1 \mathrm{U} \mathrm{HiFi}$ Taq DNA polymerase (Transgene, Beijing, China). All competitive PCR were performed in a total volume of $25 \mu \mathrm{L}$ and cycled in a PTC-100 Peltier Thermal Cycler (MJ Research Bio-Rad) with an overlay of mineral oil. The thermal cycle conditions were as follows: $94^{\circ} \mathrm{C}$ for $5 \mathrm{~min}$ in the initial step, then 25 cycles of $94^{\circ} \mathrm{C}$ for $30 \mathrm{~s}, 54^{\circ} \mathrm{C}$ for $20 \mathrm{~s}$, and $72^{\circ} \mathrm{C}$ for $20 \mathrm{~s}$, followed by $72^{\circ} \mathrm{C}$ for $5 \mathrm{~min}$ for a final extension.

\section{Southern blotting hybridization}

Genomic DNA was obtained by the same method as for competitive PCR. Genomic DNA (60-100 $\mu \mathrm{g}$ ) was digested with HindIII (TaKaRa, Dalian, China) for $24 \mathrm{~h}$. The digested DNA was separated by electrophoresis on a $0.8 \%$ agarose gel for $12-14 \mathrm{~h}$ at $20 \mathrm{~V}$. After depurinating in 0.25 $\mathrm{N} \mathrm{HCl}$, DNA fragments were transferred to a positive nylon membrane (Millipore Corporation, USA). Southern blotting analysis was performed using a DIG-High Prime DNA Labeling and Detection Starter Kit I (Roche, Mannheim, Germany) following the manufacturer instructions. A $0.45-\mathrm{kb}$ DNA fragment of the hygromycin gene was used as the probe.

\section{RESULTS}

\section{Principle of the determination of transgene copy number}

The physical map of pSSRCopy is described in Figure 1. Hygromycin was used as the selection agent in plant transformation, and GUS gene was the primary judge for the positive transgene lines. The SSR locus 'PT1199' is a homogenote in diploid tobacco K326 (Bindler et al., 2007). If a single copy of pSSRCopy is integrated into the genome of K326, only one of the two allelic chromosomes harbors the transgene. Accordingly, the band of SSR-95 is expected to be half as dense as the band of SSR-168. If two copies of pSSRCopy are integrated into the genome of K326, the band of SSR-95 is expected to be as dense as the band of SSR-168. If multiple copies of pSSRCopy are integrated into the genome of K326, the density of the band of SSR-95 is more than the density of the band of SSR-168. This principle is illustrated in Figure 2. 


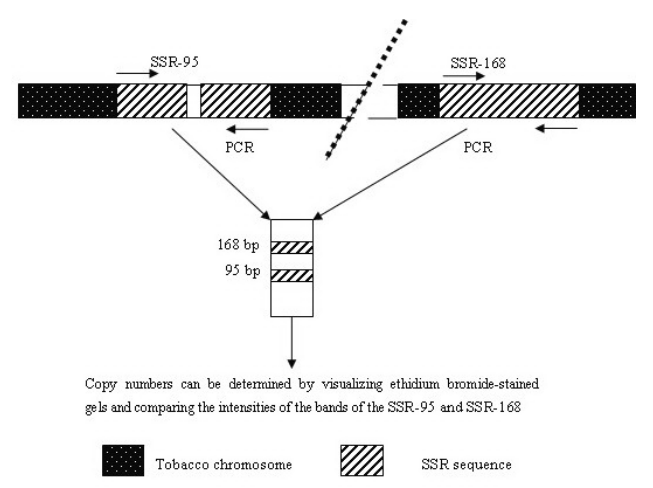

Figure 2. Principle of the determination of transgene copy number in tobacco. The short horizontal arrows show the primer-binding sites. The diagonal rectangles represent the simple sequence repeat (SSR) sequence and the black rectangles with dot represent the tobacco chromosome.

\section{Analysis of transgene copy number using competitive PCR}

Genomic DNA was isolated by the CTAB method and was diluted to a concentration of $50 \mathrm{ng} / \mu \mathrm{L}$ with double-distilled water. Competitive PCR was performed using the primers of the SSR locus PT1199. As shown in Figure 3, the 95-bp lower band is half as dense as the 168-bp upper band in transgenic tobacco lines A, C, and D, indicating the presence of a single transgene copy. In line B, the lower band is denser than the upper band, indicating the presence of more than two transgene copies.

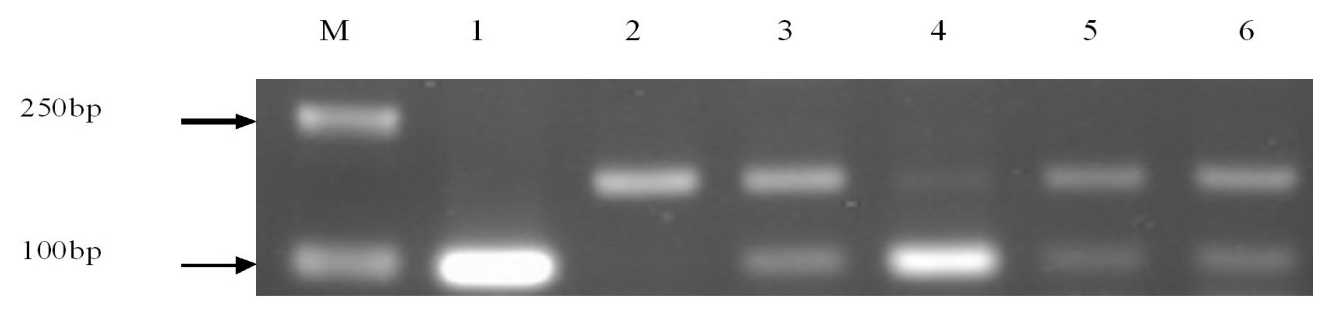

Figure 3. Detection of competitive PCR product of tobacco transgenic lines on a $2 \%$ agarose gel. $\mathrm{M}=\mathrm{DL} 2000$ DNA marker (Transgene, Beijing, China); lane $1=$ positive control (pSSRCopy plasmid); lane 2 = negative control (the DNA of non-transgenic tobacco); lane $3=$ line $\mathrm{A}$; lane $4=$ line $\mathrm{B}$; lane $5=$ line $\mathrm{C}$; lane $6=$ line $\mathrm{D}$.

\section{Southern blotting analysis}

Southern blotting analysis was carried out to confirm the reliability of competitive PCR results. Genomic DNA was digested by the restriction enzyme HindIII, which has a unique site in the T-border region of plasmid pSSRCopy. A single transgene copy is present in lines A, C, and D, and more than two copies are present in line B (Figure 4). These results are consistent with PCR results for these transgenic tobacco lines. 


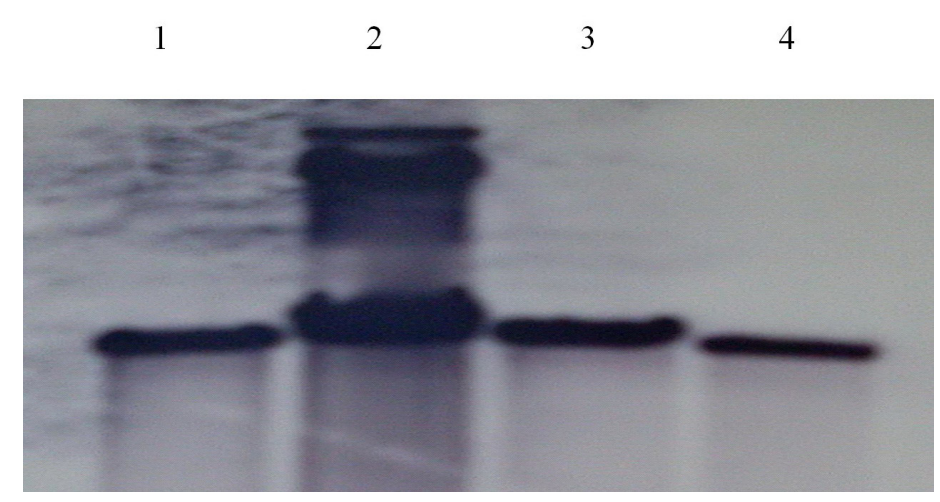

Figure 4. Southern blotting analysis of transgenic tobacco lines. Lane $1=$ line A; lane $2=$ line $\mathrm{B}$; lane $3=$ line $\mathrm{C}$; lane $4=$ line $\mathrm{D}$.

\section{DISCUSSION}

Tobacco is widely used in biotechnology and functional genomics. Transgenic plants with one or two transgene copies are generally preferred for stable and high-level expression of the transgene (Yi et al., 2008). The Southern blotting method is the most commonly used for copy number determination but is time-consuming and labor-intensive. Our rapid, straightforward, and inexpensive method is useful for functional genomics and large-scale projects related to tobacco biotechnology.

Another advantage of our method is that transgene copy number can be determined using a small amount of tobacco leaf tissue. Thus, only a small portion of a tobacco leaf from each young seedling is needed for the analysis. Therefore, only the transgenic lines with the single transgene copy need to be trained into the mature plants for the physiological and genetic analysis, thus reducing labor and storage space.

In our new method, the plasmid pSSRCopy contained an SSR locus of tobacco. It is widely accepted that SSRs isolated from a source genome can be transferred to different individuals in the same species and genus, even in the same family. Yamamoto et al. (2001) successfully transferred SSR primers derived from apple to pear. Therefore, the plasmid pSSRCopy that we constructed maybe can be used for other transgenic Solanaceae plants for the determination of transgene copy number.

In conclusion, our new method based on competitive PCR is simple, rapid and inexpensive. The determination of transgene copy number can be performed by standard PCR using a small portion of a leaf from young transgenic plants.

\section{ACKNOWLEDGMENTS}

Research supported by the National Science Foundation of China (\#30671438), the National "863" Project of Key Transgenic Program of the Ministry of Science and Technology of China (\#2008AA10Z157), and a Science and Technology Support Plan of Jiangsu Province of China (\#BE2008316). 


\section{REFERENCES}

Bindler G, van der Hoeven R, Gunduz I, Plieske J, et al. (2007). A microsatellite marker based linkage map of tobacco. Theor. Appl. Genet. 114: 341-349.

Hofgen R and Willmitzer L (1988). Storage of competent cells for Agrobacterium transformation. Nucleic Acids Res. 16: 9877.

Horsch RB, Fry JE, Hoffmann NL, Eichholtz D, et al. (1985). A Simple and General Method for Transferring Genes into Plants. Sci. 227: 1229-1231.

Kumpatla SP, Teng W, Buchholz WG and Hall TC (1997). Epigenetic transcriptional silencing and 5-azacytidine-mediated reactivation of a complex transgene in rice. Plant Physiol. 115: 361-373.

Mason G, Provero P, Vaira AM and Accotto GP (2002). Estimating the number of integrations in transformed plants by quantitative real-time PCR. BMC Biotechnol. 2: 20.

Murray MG and Thompson WF (1980). Rapid isolation of high molecular weight plant DNA. Nucleic Acids Res. 8: 43214325.

Yamamoto T, Kimura T, Sawamura Y, Kotobuki K, et al. (2001). SSRs isolated from apple can identify polymorphism and genetic diversity in pear. Theor. Appl. Genet. 102: 865-870.

Yang L, Ding J, Zhang C, Jia J, et al. (2005). Estimating the copy number of transgenes in transformed rice by real-time quantitative PCR. Plant Cell Rep. 23: 759-763.

Yi CX, Zhang J, Chan KM, Liu XK, et al. (2008). Quantitative real-time PCR assay to detect transgene copy number in cotton (Gossypium hirsutum). Anal. Biochem. 375: 150-152. 